\title{
Acquired uterine arteriovenous fistula due to a previous cornual pregnancy with placenta accreta: A case report
}

\author{
DONGDONG GUAN ${ }^{1}$, JIE WANG $^{2}$, LIJU ZONG $^{2}$, SHAN LI $^{2}$ and YOU-ZHONG ZHANG ${ }^{2}$ \\ ${ }^{1}$ Department of Obstetrics and Gynecology, The Affiliated Hospital of Binzhou Medical University, \\ Binzhou, Shandong 256603; ${ }^{2}$ Department of Gynecology and Obstetrics, Qilu Hospital, \\ Shandong University, Jinan, Shandong 250012, P.R. China
}

Received March 24, 2015; Accepted April 18, 2016

DOI: $10.3892 /$ etm.2017.4354

\begin{abstract}
The present report describes a case of an acquired uterine arteriovenous fistula, which, following surgery and postoperative pathological analysis, was confirmed as a previous cornual pregnancy with placenta accreta. The patient was a 37-year-old woman (gravida 3; para 2) who had previously delivered two children via cesarean section (in 2004 and 2010, respectively) and also had a spontaneous abortion (2008). She had experienced continuous menstrual bleeding for $\sim 20$ days and had a history of prolonged ( 30 days) vaginal bleeding 13 months earlier. Other pregnancy-related diseases were excluded following a negative serum human chorionic gonadotropin test, and the diagnosis was confirmed by Doppler ultrasonography. An emergency hysterectomy was subsequently performed due to the large amount of vaginal bleeding. Postoperative pathology confirmed the uterine arteriovenous malformation and demonstrated that the cause was a previous cornual pregnancy with placental implantation. The patient successfully recovered following the surgery.
\end{abstract}

\section{Introduction}

Uterine arteriovenous fistula (AVF) is generally divided into two types, namely congenital uterine AVF and acquired AVF $(1,2)$. Congenital uterine AVF arises from original embryonic vascular structures due to developmental abnormalities or cessation, and often involves multiple vessel traffic and other nearby organs. Acquired AVF can be caused by major trauma (including surgery, childbirth, all types of abortion and

Correspondence to: Professor You-Zhong Zhang, Department of Gynecology and Obstetrics, Qilu Hospital, Shandong University, 107 West Wenhua Road, Jinan, Shandong 250012, P.R. China E-mail: zhangyouzhong@vip.sina.com

Abbreviations: AVF, arteriovenous fistula; hCG, human chorionic gonadotropin

Key words: uterine arteriovenous fistula, acquired, old cornual pregnancy, placenta accreta, vaginal bleeding curettage), infection and tumors, particularly trophoblastic tumors, and is frequently a single arteriovenous communication not involving the surrounding tissue (3-5). Trophoblastic tumors have an affinity for vasculature, and readily erodable blood vessels; chemotherapy may damage the blood vessel walls, causing the formation of arterial and venous traffic branches or uterine arteriovenous fistula formation $(2,6)$. The disease incidence of uterine AVF is a very low, and accurate statistics are lacking. The treatment methods include uterine artery embolization and uterine surgery. When conducting an interventional radiology consultation, angiographic uterine artery embolization is recommended as the first choice for preserving the fertility of young patients with uterine AVFs $(7,8)$.

The present case report describes a patient with an acquired uterine AVF due to a previous cornual pregnancy with placenta accreta.

\section{Case report}

A 37-year-old woman (gravida 3; para 2) was admitted to Qilu Hospital (Jinan, China) on September 10, 2013 because of continuous menstrual bleeding lasting 20 days. She had previously delivered two children via cesarean section in 2004 and 2010, respectively, and experienced a spontaneous abortion in 2008 with dilatation and curettage. She reported regular menstruation cycles and no obvious menopausal history prior to the onset of these symptoms. The patient's last menstrual period began on August 20, 2013; it was regular and lasted 5 days. Her symptoms were sometimes exacerbated by dizzinesss and fatigue, although she denied abdominal pain. Color Doppler ultrasonography (Philips iU22; Philips Medical Systems, Inc., Bothell, WA, USA), was performed, which exhibited heterogeneity at the left uterine cornu that was considered to be an AVF (Fig. 1). Subsequently, a serum human chorionic gonadotropin (hCG) test was completed, the results of which confirmed that the patient was not pregnant (hCG concentration, <0.60 IU/1). The patient was immediately treated with two units of packed red blood cells for anemia (hemoglobin concentration, $65 \mathrm{~g} / \mathrm{l}$; normal adult blood reference standard, 110-160 g/l). Following blood transfusion the symptoms of vaginal bleeding were reduced. The patient 


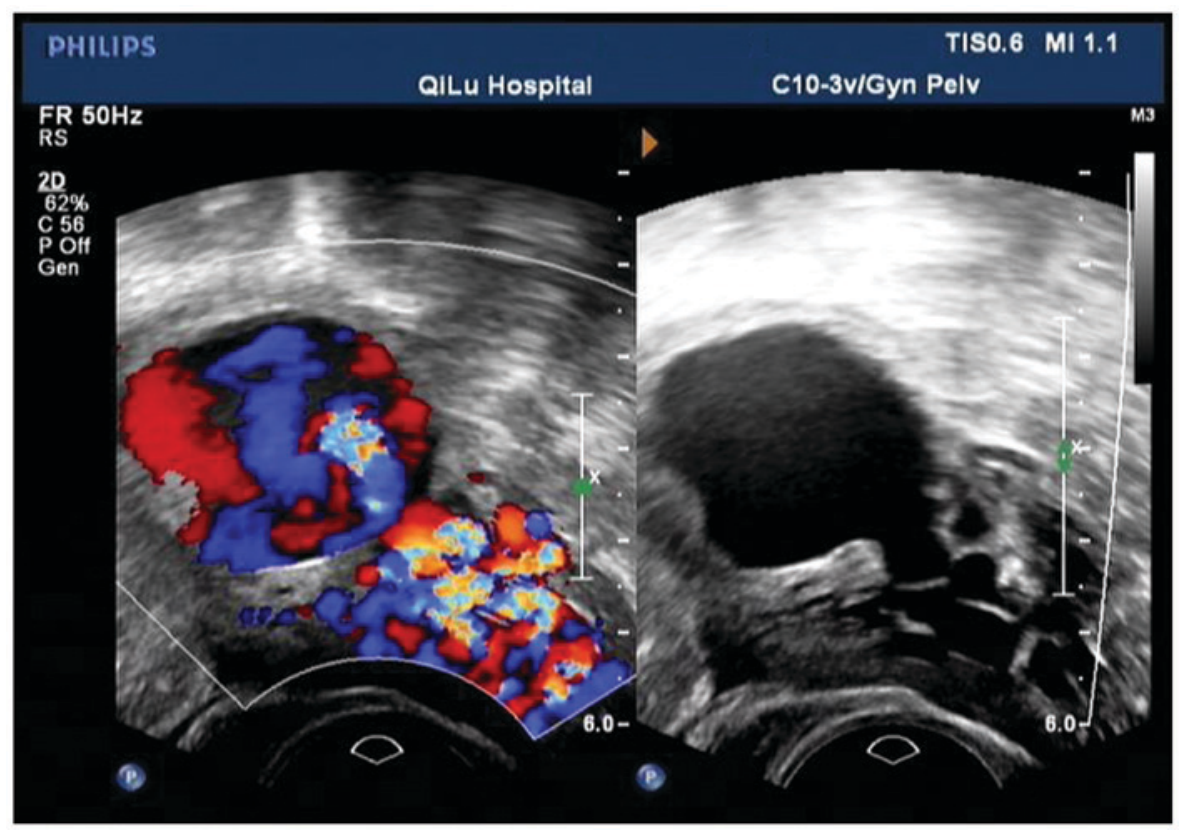

Figure 1. Color Doppler ultrasonography of a longitudinal section of the patient's uterus. The vessels of the left uterine tube were twisted (width, $0.5-0.6 \mathrm{~cm}$ ) and were partially connected with the left uterine horn, as detected by the blood imaging.

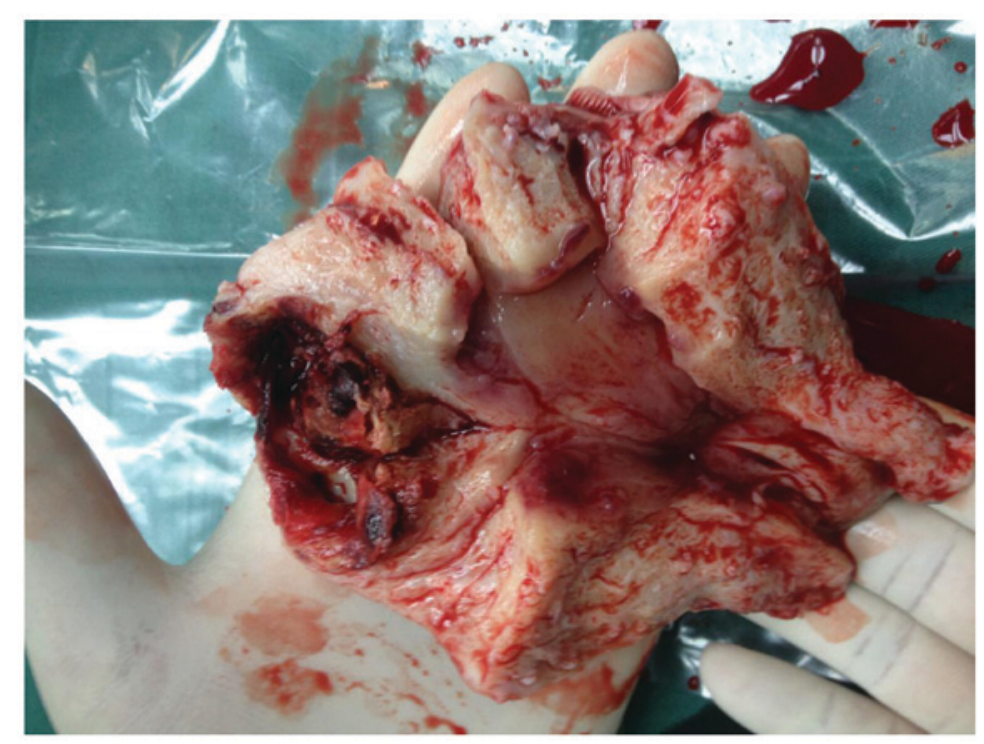

Figure 2. Postoperative uterine specimen. An obvious cystic cavity was detected in the left uterine tube which was protruding and connected with the uterine cavity via a blood clot with necrotizing tissues inside.

was then admitted to the gynecological ward of the hospital due to the diagnosis of uterine AVF, and a hysterectomy was planned as the patient expressed no desire to preserve her fertility.

On the morning of September 12, the patient experienced extensive vaginal blood loss $(\sim 400 \mathrm{ml})$ and presented with the clinical symptoms of mentality indifference and poor vital signs (blood pressure, $80 / 50 \mathrm{mmHg}$; oxygen saturation, $95 \%$ ). The patient was prepared for emergency surgery while recovering from shock. A 500-ml dose of hydroxyethyl starch (Shandong Weigao Group, Weihai, China) was administered for rapid rehydration therapy. Intraoperative findings demonstrated that the anterior uterus was slightly larger than normal, and the left uterine horn was convex, with dimensions of $4 \times 3 \times 3 \mathrm{~cm}$, purplish blue in color, with expansive and tortuous vessels to the broad ligament, whereas the bilateral ovarian and fallopian tubes were normal. As the patient was in a state of shock and hemoglobin concentration and blood gas analysis could not be detected, 4 units of packed red blood cells and $400 \mathrm{ml}$ plasma were transfused during the surgery. The uterus was excised was sectioned, which demonstrated a protruding cavity with old blood in the left uterine horn, whereas the endometrium of the uterus and the cervical canal were normal (Fig. 2). Analysis of the intraoperative sections demonstrated an expansive cystic cavity, sphacelus, blood clots and the subsidiary uterine AVF. 


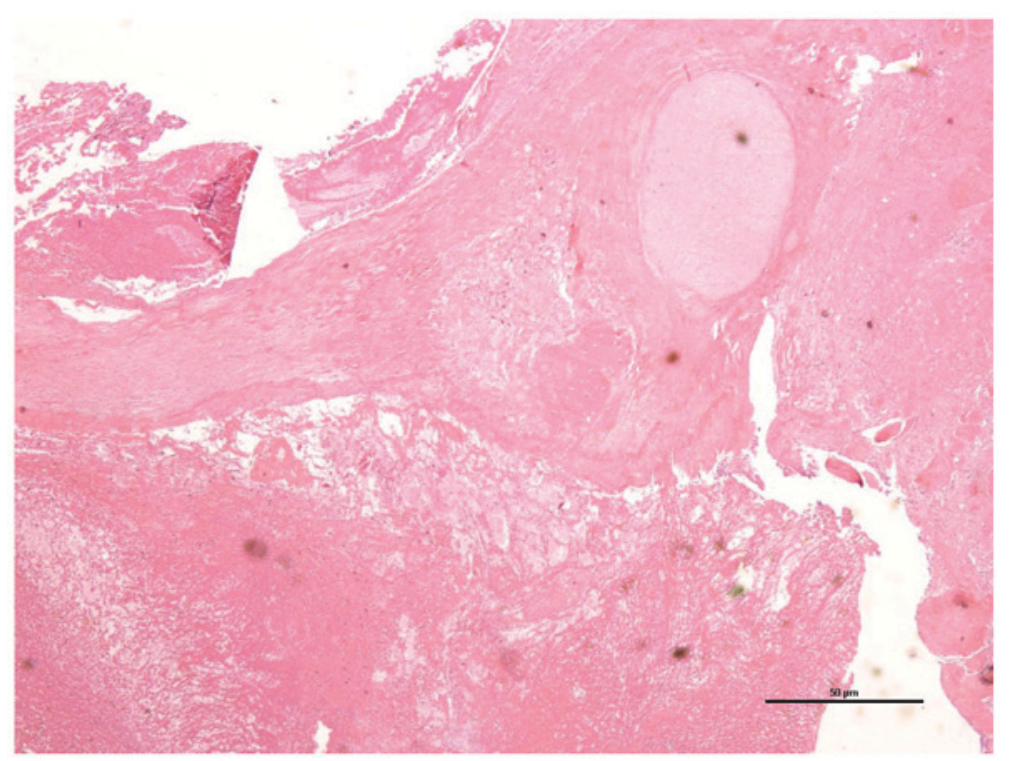

Figure 3. Paraffin-embedded sections were analyzed, which demonstrated fibrosis of the placental villi and a small amount of cartilage components, which were considered to be residual fetal components, in the myometrium (scale bar, $50 \mu \mathrm{m}$; magnification, $\mathrm{x} 200$; hematoxylin and eosin staining).

Postoperatively, sections were paraffin-embedded and subsequent analysis demonstrated fibrosis of the placental villi with placenta accreta and a small amount of cartilage components, which were considered to be residual fetal components, were found in the uterine muscle layer during the proliferative phase of the endometrium (Fig. 3). There were no intraoperative or postoperative complications. The patient recovered well, her symptoms were cured 6 days after surgery and she was no longer in discomfort 1 month post- surgery.

Further details about the patient's history prior to the surgery were disclosed following the surgery. Two years previously, the patient underwent bilateral tubal surgery with an adhesive agent; however, the left fallopian tube remained obstructed. Following this, the patient did not take any contraceptive medication. However, 13 months ago the patient experienced irregular vaginal bleeding for $\sim 30$ days without previously delayed menstruation or expulsion of tissue in vaginal blood. Over the next 6 months the patient experienced amenorrhea without an early pregnancy response. Following 4 months of amenorrhea, she accepted medroxyprogesterone (12 mg once daily for 7 days) to adjust her menstrual cycle as her pregnancy test was negative. Color Doppler ultrasonography was performed, which ruled out the possibility of pregnancy. Two months later her menstruation recovered and the patient experienced regular menstrual cycles for 7 months until the disease symptoms presented.

\section{Discussion}

To the best of our knowledge, the present case report is the first to describe a patient with an acquired uterine AVF due to a previous cornual pregnancy with placenta accreta. AVF, which is also known as arteriovenous malformation (AVM), refers to an abnormal passage between the uterine artery and vein, which results in artery blood flowing directly into a vein (9). Congenital AVFs, which are extremely rare, are caused by dysplasia of the embryonic vascular structures and are often complicated with pelvic vascular malformation. Acquired AVFs often result in trauma to the uterus, due to dilation and curettage, infection, gestational trophoblastic disease or endometrial carcinoma (1). The predominant pathological changes include multiple small arteriovenous fistula between the traumatized arterial branches and muscular venous (10). Notably, the development of acquired AVF is slower, therefore the symptoms appear a long time after the uterine curettage or cesarean section occurred. The most common symptoms of AVF are abnormal and heavy vaginal bleeding, which manifests as hypermenorrhea or metrorraghia induced by blood vessel malformation exposure and rupture, dyspareunia and pelvic pain (3). The present patient should be considered as an acquired arteriovenous fistula as, although she had a history of cesarean section $(n=2)$ and curettage $(n-1)$, the uterus arteriovenous fistula remains away from the uterine scar and was in the left uterine horn (Fig. 3). It seems that has nothing to do with it. Yet the patient had undergone a failed tubal blockage surgery, which may be the cause of the abnormal cornual pregnancy with placenta accreta and subsequent incomplete abortion, of which the patient was unaware; thus, the AVF was formed. Kim et al (11) have previously reported the case of 20 -week pregnancy uterine arteriovenous fistula with placenta accreta. Furthermore, Corusic et al (6) have previously described a patient with uterine arteriovenous fistula caused by residual placenta following choriocarcinoma. These cases are similar to the present case, in that they were not diagnosed early.

Doppler ultrasonography is the most commonly used method used to diagnose AVF, due to its convenience and efficiency (7). The ultrasound images captured of the present patient (Fig. 1) demonstrated the typical characteristics of uterine arteriovenous fistula: Oval or irregular no echo area with flowing punctiform and low or weak echo; and in the honeycomb anechoic area, saccate mixed solid and tubulose interpenetration, with a multidirectional flow and apparent reverse flow due to aliasing. Color Doppler flow imaging (CDFI) displays the signal of blood flow as a colorful mosaic, 
as the red and blue are mixed as color aliasing in lesions. In CDFI analysis, AVF appears as an intensely vascular tangle that exhibits a flow with high velocity and low resistance. However, there are limitations to the use of ultrasound in these circumstances as it is difficult to distinguish AVF from other diseases that are associated with pregnancy, including incomplete abortion and trophoblastic diseases, particularly in intrauterine embryo residues, residues and the myometrium, which can also exhibit rich blood flow signals $(2,12-14)$. Therefore, in the present case, experienced ultrasound clinicians cross-referenced the CDFI findings with the patient's blood $\beta$-hCG, which were determined to be negative after testing 2 times, and she was diagnosed with uterine arteriovenous fistula. Although further pelvic angiography is the gold standard for the diagnosis of AVF, it was not necessary in the present case. Postoperative pathology confirmed the uterine AVM and simultaneously demonstrated that the cause was a previous cornual pregnancy with placental implantation.

Although uterine arteriovenous fistula remain rare, they can cause irregular or heavy vaginal bleeding which may endanger the patient's life $(1,14)$. Clinically, when patients present with unexplained recurrent vaginal bleeding, curettage should not be immediately performed (14); treatment should be planned according to the patients' symptoms, arteriovenous fistula location and whether they wish to preserve fertility. Selective arterial embolization is the most commonly used method of clinically conservative treatment, which is repeatable and has a success rate of $\sim 90 \%(4,8,15)$. In addition, laparoscopic surgery has shown its superiority. Corusic et al (6) reported a case of an acquired large AVM due to choriocarcinoma that was successfully treated with laparoscopic surgery by removing the AVM, laparoscopic bipolar coagulation of the uterine vessels and ligation of the uterine artery. Furthermore, Levy-Zaubermann et al (5) reported a case of AVF in which the patient underwent ligation of the internal iliac artery via laparoscopic surgery. However, when patients exhibit embolization failure, repeated hemorrhage, are difficult to follow-up and demonstrate no fertility wishes, hysterectomy is an effective treatment. In the present case, the patient consented to hysterectomy and the surgery was timely and successful.

Although there are various reports on uterine arteriovenous fistula cases $(1,3,9-11)$, to the best of our knowledge, the present study is the first to report a case of uterine arteriovenous fistula due to an old cornual pregnancy with placenta accreta which was accompanied by a history of tubal surgery, sustained normal menstruation prior to onset and negative
$\beta$-hCG. Therefore, we recommend that detailed a patient case history is taken in order to achieve a more accurate clinical diagnosis and effective treatment.

\section{References}

1. Halperin R, Schneider D, Maymon R, Peer A, Pansky M and Herman A: Arteriovenous malformation after uterine curettage: A report of 3 cases. J Reprod Med 52: 445-449, 2007.

2. Timmerman D, Wauters J, Van Calenbergh S, Van Schoubroeck D, Maleux G, Van Den Bosch T and Spitz B: Color Doppler imaging is a valuable tool for the diagnosis and management of uterine vascular malformation. Ultrasound Obstet Gynecol 21: 570-577, 2003.

3. Nasu K, Fujisawa K, Yoshimatsu J and Miyakawa I: Uterine arteriovenous mlformation: Ultrasonographic, magnetic resonance and radiogical findings. Gynecol Obstet Invest 53: 191-194, 2002.

4. O'Brien P, Neyastani A and Buckley AR: Uterine arteriovenous malformations: From diagnosis to treatment. J Ultrasound Med 25: 1387-1392; quiz 1394-1395, 2006.

5. Levy-Zaubermann Y, Capmas P, Legendre G and Fernandez H: Laparoscopic management of uterine arteriovenous malformation via occlusion of internal iliac arteries. J Minim Invasive Gynecol 19: 785-788, 2012.

6. Corusic A, Barisic D, Lovric H, Despot A and Planinic P: Successful laparoscopic bipolar coagulation of a large arteriovenous malformation due to invasive trophoblastic disease: A case report. J Minim Invasive Gynecol 16: 368-371, 2009.

7. Valenzano M, Foglia G, Tedeschi C, Paoletti R and Fulcheri E: Color Doppler sonography of uterine arteriovenous malformation. J Clin Ultrasound 28: 146-149, 2000.

8. Ghai S, Rajan DK, Asch MR, Muradali D, Simons ME and TerBrugge KG: Efficacy of embolization in traumatic uterine vascular malformations. J Vasc Interv Radiol 14: 1401-1408, 2003.

9. Yang JJ, Xiang Y, Wan XR, Gao Y, Cheng NH, Liu W, Yang N and Yang XY: Clinical analysis of uterine arteriovenous fistula caused by vaginal bleeding. Zhonghua Fu Chan $\mathrm{Ke} \mathrm{Za}$ Zhi 39: 797-800, 2004 (In Chinese).

10. Takeda A, Koyama K, Imoto S, Mori M, Sakai K and Nakamura H: Progressive formation of uterine arteriovenous fistula after laparoscopic assisted myomectomy. Arch Gynecol Obstet 280: 663-667, 2009.

11. Kim TH, Lee HH and Kwak JJ: Conservative management of abnormally invasive placenta: Choriocarcinoma with uterine arteriovenous fistula from remnant invasive placenta. Acta Obstet Gynecol Scand 92: 988-990, 2013.

12. Sanguin S, Lanta-Delmas S, Le Branche A, Grardel-Chambenoit E, Merviel P, Gondry J and Fauvet R: Uterine arteriovenous malformations: Diagnosis and treatment in 2011. Gynecol Obstet Fertil 39: 722-727, 2011.

13. Grivell RM, Reid KM and Mellor A: Uterine arteriovenous malformations: A review of the current literature. Obstet Gynecol Surv 60: 761-767, 2005.

14. Yahi-Mountasser H, Collinet P, Nayama M, Boukerrou M, Robert Y and Deruelle P: Intrauterine arteriovenous malformations. J Gynecol Obstet Biol Reprod (Paris) 35: 614-620, 2006 (In French).

15. Lim AK, Agarwal R, Seckl MJ, Newlands ES, Barrett NK and Mitchell AW: Embolization of bleeding residual uterine vascular malformations in patients with treated gestational trophoblastic tumors. Radiology 222: 640-644, 2002. 